\title{
UTILIZAÇÃO DAS FERRAMENTAS DE ABORDAGEM FAMILIAR COMO SUBSÍDIO PARA O CUIDADO MULTIPROFISSIONAL NO ÂMBITO DA ESTRATÉGIA SAÚDE DA FAMÍLIA
}

\author{
USE OF FAMILY APPROACH TOOLS AS A SUBSIDY FOR \\ MULTIPROFESSIONAL CARE IN THE FRAMEWORK OF THE FAMILY \\ HEALTH STRATEGY
}

\begin{abstract}
Fernanda Antônia Jesus, Jean Alfredo Albuquerque, Vanelle Rodrigues Fonseca, Carla Patrícia Martins Cardoso
\end{abstract}

Universidade Estadual de Montes Claros - UNIMONTES

\begin{abstract}
The Family Health Strategy addresses the family as a center of care, considering its physical, biological, social and cultural characteristics. The family approach tools promote the rapprochement between health professionals and families, in addition to providing an understanding of the functioning of family members and their relationships. The objective of the present study was to describe the use of family approach tools as part of the situational diagnostic process of a family registered in the area of comprehension. This is a descriptive case study of a qualitative methodological approach carried out in the period of October from 2018 to March 2019, by a multiprofessional team, using the following familiar approach tools: Genogram, Family Life Cycle, FIRO, PRACTICE and Family Conference. It was possible to identify in family settings and relationships the main problems and diseases prevalent in the family. The use of familiar tools provided the knowledge about the particularities of family dynamics, allowing the development of interventions appropriate to the family reality. The family health team assumes an important role with families and is a point of support in relation to the health / illness issues and conflicts experienced by them.
\end{abstract}

Key words: Primary Health Care; Family Health Strategy; Family Dynamics; Chronicdiseases.
Resumo

A Estratégia Saúde da Família aborda a família como um centro de cuidado, considerando suas características físicas, biológicas, sociais $e$ culturais. As ferramentas de abordagem familiar promovem a aproximação entre os profissionais de saúde $e$ as famílias, além de possibilitar a compreensão do funcionamento dos membros familiares e suas relações. O objetivo do presente estudo foi descrever a utilização das ferramentas de abordagem familiar como parte do processo diagnóstico situacional de uma família cadastrada na área de abrangência.Trata-se de um estudo de caso, descritivo, de abordagem metodológica qualitativa, realizado no período de outubro de 2018 a março de 2019, por uma equipe multiprofissional, com utilização das seguintes ferramentas de abordagem familiar: Genograma, Ciclo de Vida Familiar, FIRO, P.R.A.C.T.I.C.E. e Conferência Familiar. Foi possivel identificar nas configurações e relações familiares os principais problemas e as doenças prevalentes na família. $O$ uso das ferramentas de abordagem familiar propiciou o conhecimento acerca das particularidades da dinâmica da família, permitindo assim o desenvolvimento de intervenções adequadas à realidade familiar. A equipe de saúde da família assume um importante papel junto às famílias e configura-se como ponto de apoio no que diz respeito às questões de saúde/doença e conflitos vivenciados pelas mesmas.

Palavras chave: Atenção Primária à Saúde; Estratégia Saúde da Família; Dinâmica Familiar; Doenças Crônicas. 
Introdução

A Estratégia Saúde da Família (ESF) surge como uma política de reorganização da Atenção Primária no Brasil, implementando ações de promoção, proteção e recuperação da saúde, de forma integral e continuada. Tais ações são embasadas no reconhecimento das necessidades da população atendida e identificadas através dos vínculos criados e fortalecidos entre os usuários dos serviços e os profissionais de saúde em contato com o território ${ }^{1}$.

Nesse contexto, a ESF aborda a família como um centro de cuidado, considerando as suas características físicas, biológicas, sociais e culturais. É, pois, imprescindível que os profissionais de saúde atuantes na Atenção Primária compreendam o conjunto familiar, para, assim, planejar e implementar ações voltadas para solucionar os entraves identificados.

Destaca-se que a dinâmica e as relações familiares podem interferir no processo saúdedoença de seus integrantes. A partir do momento em que a família oferece aos seus membros cuidado, afetividade, atenção e carinho, ela passa a funcionar como uma importante fonte de suporte que será indispensável para o desenvolvimento saudável de seus membros ${ }^{2-3}$.

Dessa forma, torna-se necessária a realização do estudo da família para conhecer e compreender os impactos causados pela interação familiar nas condições de saúde. Para tanto, algumas ferramentas de abordagem familiar são utilizadas, como por exemplo: Genograma, Ciclo de Vida Familiar, FIRO, P.R.A.C.T.I.C.E. e a Conferência Familiar. Tais ferramentas têm como objetivo promover a aproximação nas relações entre profissionais e famílias, buscando a compreensão do funcionamento do sujeito e suas relações ${ }^{4,5}$.

Este estudo justifica-se pela importância que o conjunto familiar apresenta na busca da compreensão do modo como o sujeito se relaciona em seu contexto de vida ${ }^{6}$. Pode-se inferir que a família exerce influência direta no bem estar, na cultura e nas condições de saúde dos seus membros. Assim, torna-se imprescindível conhecer a composição e a dinâmica da estrutura familiar, bem como os problemas de saúde e as vulnerabilidades que se apresentam, para assim planejar e implementar ações específicas para cada grupo familiar.

Nesse sentido, o trabalho tem como objetivo descrever a utilização das ferramentas de abordagem familiar como parte do processo diagnóstico situacional de uma família cadastrada na área de abrangência.

\section{Metodologia}

Trata-se de um estudo qualitativo, descritivo, realizado no período de outubro de 2018 a março de 2019 pela equipe multiprofissional de uma ESF composta por uma enfermeira, uma psicóloga e um cirurgiãodentista, integrantes de um programa de residência multiprofissional em saúde da família, na cidade de Montes Claros-MG. O território de abrangência da ESF está localizado na zona urbana da cidade de Montes Claros e atende aproximadamente a 2.612 indivíduos distribuídos em 802 famílias.

Para a escolha da família foram estabelecidos os seguintes critérios de inclusão: ser cadastrada na ESF; apresentar algum problema de saúde e/ou conflito familiar que viabilizasse a aplicação dos instrumentos de abordagem à família; ser receptiva com a equipe e com as intervenções propostas.

Para a realização deste estudo foi selecionada uma família indicada pelos agentes comunitários de saúde depois da observação de que a paciente índice comparecia semanalmente ao grupo hiperdia, apresentando sempre valores pressóricos e glicêmicos descompensados, mesmo com o relato de utilização correta das medicações hipoglicêmicas e anti-hipertensivas.

Para coleta de informações foi realizado um total de sete visitas ao domicílio da família, com duração média de 50 minutos. As duas visitas iniciais foram realizadas com a finalidade de explicar o objetivo do estudo da família, esclarecer os termos da participação e verificar o interesse dos membros da família quanto a sua participação e as visitas subsequentes foram para aplicação das ferramentas de abordagem familiar e para a instituição do plano de cuidados.

$O$ estudo se pautou nas normas da resolução 466/12, do Conselho Nacional de Saúde $^{7}$ sobre pesquisas envolvendo seres humanos. Foi aprovado pelo Comitê de Ética em Pesquisa da Universidade de Montes Claros, sob parecer $\mathrm{n}$-572.244. Os nomes dos membros da família são fictícios, no intuito de preservar o anonimato.

\section{Resultados e Discussão}

A família em estudo reside em uma área de invasão de terreno privado em um bairro periférico, na cidade de Montes Claros-MG. O domicílio construído de alvenaria possui cinco cômodos, varanda e uma área externa, dividida para lavanderia e armazenamento dos materiais recicláveis. A rua de acesso à residência da 
família não é asfaltada, a infraestrutura do domicilio é precária, a energia elétrica e o abastecimento de água não ocorrem de maneira legalizada.

A paciente índice é a senhora (Sra.) Júlia, 55 anos, católica, hipertensa, diabética, casada há 35 anos com o Senhor (Sr.) Luiz, 57 anos, hipertenso e em uso abusivo de álcool. Ambos têm como atividade laboral a coleta e venda de materiais recicláveis.

O diagnóstico de hipertensão arterial sistêmica (HAS) da paciente índice ocorreu há 30 anos, durante sua primeira gestação. Já o diagnóstico do diabetes mellitus ocorreu há cerca de 10 anos, em consequência de uma lesão na falange distal do segundo dedo que resultou em amputação da mesma.

As elevações constantes dos níveis pressóricos e glicêmicos da paciente índice configuraram o ponto chave para escolha da família para este estudo. Acrescenta-se que a Sra. Júlia é analfabeta, por esse motivo o agente comunitário presta-lhe auxílio, organizando as medicações da semana em potes, identificando o horário de ingestão com símbolos de sol, prato de refeição e lua correspondentes à manhã, ao almoço e à noite, respectivamente.

Nesse sentido, foi possível que a equipe de saúde verificasse que os problemas que interferem na condição de saúde da paciente índice não eram apenas de caráter individual, motivo pelo qual notou-se a necessidade de uma intervenção familiar para uma possível coresponsabilização da família quanto aos cuidados devidos a Sra. Júlia. Para tanto, foram utilizadas as ferramentas de abordagem familiar que serão descritas a seguir.

\section{Genograma}

Utilizado para a construção da configuração gráfica da estrutura familiar, o genograma possibilita a representação dos diferentes membros da família, bem como as principais patologias, as diversas relações e os principais hábitos entre os membros familiares ${ }^{4}$.

Figura 1. Genograma da família em estudo, realizado em dezembro de 2018.



Conforme percebido na Figura 01, o casal (Sra. Júlia e Sr. Luiz) possui três filhos, sendo Carla, com 30 anos, a mais velha, separada, vendedora ambulante de roupas, teve uma união estável com Sandro e com ele teve três filhos: Carlos, 14 anos, Cosme, 11 anos e o caçula lago, sete anos; todos os filhos são estudantes. 0 segundo filho do casal é Pedro, 27 anos, que mantém uma união estável com Ivete, 26 anos, juntos tiveram um filho, Arthur, um ano de idade. A filha caçula do casal é Paula, 24 anos, trabalha com a irmã Carla com a venda de roupas.

A mãe da paciente índice, a Sra. Joana, faleceu há 52 anos devido a um quadro de eclâmpsia durante o trabalho de parto, resultando também na morte do feto cujo sexo a 
Sra. Júlia não soube informar. Consequentemente a este ocorrido, o Sr. Antônio casou-se com a Sra. Ana com quem teve cinco filhos. O mesmo veio a falecer há 32 anos vítima de cirrose. Quanto aos irmãos, a paciente ressalta que atualmente não possui contato com os mesmos, exceto com a irmã caçula.

A paciente índice relata que as filhas faziam uso de várias drogas ilícitas, provocando nela grande angústia e sofrimento. Entretanto, há cerca de dois anos, a quantidade de substâncias utilizadas foi reduzida, o que trouxe alívio para toda a família.

Em se tratando da família do Sr. Luiz, seus pais, Getúlio e Maria, tiveram 16 filhos, o Sr. Getúlio faleceu há 23 anos, em consequência de câncer e a Sra. Maria faleceu no mesmo ano, era portadora de chagas. Os pais e todos os irmãos do Sr. Luiz fazem ou já fizeram uso de álcool. Exceto a sua irmã Leide que faleceu com cinco anos de idade, devido a um quadro de Hepatite $B$.

Verificou-se que a relação familiar da Sra. Júlia e do Sr. Luiz com a filha mais velha, Carla, e os filhos da mesma é marcada por desacordo e conflito intergeracional, principalmente pelo fato da filha e netos responderem comumente de forma afrontosa ao casal.

Já a relação entre as duas filhas do casal é de proximidade e cumplicidade. No entanto, a relação do irmão Pedro com as irmãs Carla e Paula é mais distante, porém não apresenta conflitos.

Observou-se que são prevalentes na família casos de HAS, uso de álcool e relato de conflito intergeracional. A Hipertensão Arterial Sistêmica é uma condição clínica multifatorial, caracterizada por níveis elevados e sustentados de pressão arterial, possui como um dos fatores de risco a hereditariedade, ou seja, a HAS primária nos pais possui importante implicação para o surgimento dessa condição nos filhos ${ }^{8-9}$.

A família é considerada uma forte influência para o consumo da bebida alcoólica, pois é no ambiente familiar que normalmente ocorre o primeiro contato com o álcool, além disso a propagação da aceitação do uso, por parte dos pais, pode ser um fator de risco desencadeador para o uso abusivo de álcool por parte dos filhos $^{10}$.

Em se tratando do conflito intergeracional, o mesmo ocorre devido as diferenças de visão de mundo, pois o que é aceitável e correto para filhos e netos pode não ser para os pais-avós, tornando as relações familiares por vezes desarmônicas ${ }^{11}$.

O genograma permitiu identificar a estrutura genealógica familiar, as patologias de maior acometimento entre as gerações, bem como as relações estabelecidas entre os familiares. Assim, a utilização dessa ferramenta permite que o profissional atuante na estratégia de saúde da família identifique a estrutura, a dinâmica e os problemas familiares para posteriormente planejar e sugerir ações que irão contribuir para o enfrentamento das dificuldades.

\section{Ciclo de Vida Familiar}

Residem juntos, no mesmo local, a Sra. Júlia, o Sr. Luiz e os netos Carlos e Cosme. Já as filhas e o neto lago moram em frente à casa do casal e realizam todas as refeições no domicílio da paciente índice.

Verificou-se que a família em questão é caracterizada como sendo de meia idade, cujos ascendentes passaram a configurar novos papéis (marido-pai-avô; esposa-mãe-avó) ao mesmo tempo em que se prepara para a aposentadoria que proveria conforto e bem estar ao casal. Nesse estágio, alguns autores ${ }^{12}$ destacam que cabe aos profissionais de saúde promover o encorajamento dos sujeitos a realizar planos que incluam o lazer, a moradia e a aposentadoria; propor discussões acerca do processo de envelhecimento e explorar o papel de avós.

Firo

A ferramenta FIRO tem como objetivo avaliar as relações fundamentais interpessoais estabelecidas entre os membros da família. Essa ferramenta auxilia na compreensão de como as necessidades individuais afetam os relacionamentos pessoais. As características analisadas estão associadas a três necessidades interpessoais, que são: inclusão, controle e intimidade ${ }^{13}$.

A Inclusão está ligada à interação entre os membros da família, evidenciando os papeis individuais na organização. O Controle abrange as relações de poder estabelecidas, por meio das quais podemos identificar: o controle dominante, quando um exerce influência sobre todos os demais; o controle reativo, quando se estabelece reações contrárias a uma ação e controle colaborativo, quando se estabelece divisão de influências. A Intimidade refere-se às relações afetivas e ao compartilhamento de sentimentos ${ }^{14}$.

A partir da aplicação da ferramenta FIRO, na abordagem da família em questão, foram identificados conflitos quanto às necessidades interpessoais de inclusão e intimidade. No que se refere à Inclusão, observou-se a má divisão dos papéis individuais no controle das doenças de Sra. 
Júlia e Sr. Luiz. A paciente índice, por ser analfabeta, tinha dificuldade de utilizar as medicações corretamente, enquanto seu marido estava desmotivado quanto ao uso de suas medicações, uma vez que ambos não recebiam auxílio familiar. $O$ uso inadequado das medicações prejudicava o controle das condições de saúde apresentadas e foi identificado como um dos principais problemas encontrados. Quanto ao Controle, verificou-se que havia divisão de responsabilidades sobre as tomadas de decisões, o que demonstrava uma relação de poder colaborativo, com divisão de influências. No que tange à Intimidade, o que prejudicava a família e tinha repercussão negativa tanto no controle das doenças quanto na relação familiar eram os problemas afetivos entre Sra. Júlia com a filha mais velha e seus netos, além do uso abusivo de álcool por parte do Sr. Luiz que dificultava a relação conjugal em alguns momentos.

\section{P.R.A.C.T.I.C.E}

A ferramenta de abordagem familiar P.R.A.C.T.I.C.E. deriva das seguintes palavras em inglês: problem (problema), rolesandstructure (papéis e estrutura), affect (afeto), communication (comunicação), time in life (ciclo de vida), illness in Family (doênças na família), copingwith stress (lidando com o stresse), environmenorecology (meio ambiente ou ecologia). Cada palavra determina um domínio da interação familiar a ser abordado e fornece uma estrutura básica para organização das informações obtidas ${ }^{14}$.

A partir do padrão estrutural dessa ferramenta foi possível levantar as seguintes informações:

Dentro do domínio Problema, a dificuldade de controle das doenças apresentadas pela paciente índice representava o principal motivo que despertou a atuação dos profissionais de saúde. Embora a paciente se dispusesse a seguir o plano de cuidado estabelecido, não obtinha melhora e/ou controle de suas morbidades.

Quanto ao domínio Papéis e Estrutura, assim como observado na aplicação da ferramenta FIRO., existia deficiência na divisão de tarefas e responsabilidades dos membros da família, esse fator, associado à insuficiência de cuidados, agravava a situação de saúde da paciente índice.

$\mathrm{Na}$ abordagem do Afeto foi possível observar que as relações interpessoais entre os membros da família eram prejudicadas pelo uso abusivo de álcool do Sr. Luiz e pelo uso de drogas ilícitas por parte das filhas do casal.

Com relação à Comunicação, foi possível identificar deficiências, uma vez que a Sra. Júlia era totalmente responsabilizada por sua condição de saúde, não havendo a intenção de motivar a melhora, por parte dos filhos.

No que tange à fase do Ciclo de Vida, como apresentado anteriormente, verificou que se trata de uma família de meia-idade cujos ascendentes desenvolvem os papéis de pais e avós, tendo em vista que são responsáveis pelos cuidados de dois netos, resultando em uma sobrecarga de funções.

Em Doenças na Família, assim como observado no genograma, notou-se a existência de uma repetição da presença do uso de álcool e hipertensão arterial na família.

Referente ao domínio Lidando Com o Estresse, notou-se que as relações conflituosas entre a paciente índice, suas filhas e netos eram motivos de discussões e geradoras de estresse, que segundo a Sr. Júlia, dificultavam o controle de sua doença.

No último domínio, que se refere ao Meio Ambiente e Ecologia, foi possível observar que a família apresentava boa relação com o meio social de convivência que inclui o CRAS, a Igreja e a Associação de Catadores de Materiais Reclicáveis, além de um bom vínculo com os profissionais de saúde, facilitando a abordagem familiar proposta.

Essa ferramenta abrangeu todas as outras aplicadas anteriormente, facilitando a compreensão da organização familiar e dos problemas apresentados. Em tese, as informações obtidas foram relevantes para definição do plano de ação.

\section{Conferência Familiar}

A conferência familiar tem como objetivo possibilitar a intervenção multiprofissional, auxiliando em entraves cujas soluções o grupo familiar não consegue identificar, assim como possibilita promover a resolução de problemas que abrangem os membros do grupo ${ }^{15}$.

A data e horário para realização da conferência familiar foram definidos junto à família, visando a participação de todos, entretanto somente Sra. Júlia, o Sr. Luiz, a filha Paula e os profissionais de saúde, envolvidos na realização do estudo, estiveram presentes durante a realização dessa ferramenta.

Ao início da conferência, realizou-se a apresentação dos participantes, seguida pela exposição dos motivos que levaram a equipe de profissionais da saúde a escolherem a família 
para a realização do estudo. Destaca-se ainda que os objetivos e a relevância do estudo foram reafirmados, salientando, de maneira especial, as características e propósitos da conferência familiar. Posteriormente, foi realizada uma explanação sucinta e clara sobre as condições de saúde da paciente índice, enfatizando as doenças hipertensão e diabetes, reforçando a importância de cuidado permanente e contínuo, bem como as possíveis consequências da falta do tratamento.

A apresentação do genograma foi realizada de maneira explicativa, viabilizando que a família identificasse a repetição das morbidades ao longo das gerações, ao mesmo tempo em que possibilitou um espaço de conversa sobre os padrões de estilo de vida e das relações interpessoais. Em determinado momento, Paula expôs a dificuldade de auxiliar a Sra. Júlia nos cuidados demandados por suas doenças, ressaltando que a mãe possui resistência quanto à prática de atividades físicas e à manutenção da dieta, conforme orientação médica. Diante da fala da filha, a Sra. Júlia evidenciou dificuldade de adaptar sua rotina com aquilo que lhe é solicitado, uma vez que demanda mudanças em todo seu estilo de vida. Nesse sentido, foram abordadas as relações de conflitos, com foco na necessidade de diálogo e co-responsabilização entre os membros da família. Concomitantemente a esse momento, foi abordado o ciclo de vida, destacando-se a necessidade de definição dos papéis dos membros familiares e a divisão de responsabilidades.

No que se refere ao consumo de álcool do Sr. Luiz, foi possível detectar que apesar da conscientização acerca dos danos causados pela bebida no organismo, o paciente não considera o consumo realizado como abusivo e não relata desejo de interromper o uso. Dessa forma, a redução de danos do consumo de álcool foi uma estratégia apresentada à família como forma de minimizar os agravos causados à saúde.

Ao encerrar a conferência, pactuou-se com os membros presentes planos de cuidados para o enfretamento das dificuldades apresentadas. Nesse sentido, as intervençoes propostas relacionaram-se diretamente às mudanças no estilo de vida da família, como forma de prevenir agravos e manter o controle frente aos riscos inerentes de agravamento das doenças crônicas. O plano de cuidado abrangeu: redução do uso de álcool por parte do Sr. Luiz, levando em conta a estratégia de redução de danos; coresponsabilização e divisão de tarefas frente aos cuidados da Sra. Júlia e do Sr. Luiz, visando evitar a sobrecarga dos membros da família. Além disso, ressaltou-se a disponibilidade do serviço de saúde quanto ao cumprimento das metas pactuadas e a continuidade do cuidado da família. Ressaltou-se ainda a importância do repasse das informações e acordos firmados na conferência pelos participantes aos demais membros familiares.

Em consequência do número reduzido de membros familiares na conferência, os profissionais de saúde identificaram a necessidade de realizar nova reunião com a participação de maior número de integrantes do núcleo familiar. Dessa forma, uma semana após o momento de conferência familiar, os profissionais retornaram à casa da família, realizando reunião com a Sra. Júlia, Sr. Luiz, a filha Paula e a nora Ivete. Ocasião em que a equipe de saúde disponibilizou um suporte para acondicionar os potes de medicações separadamente, de cordo com os turnos corretos de utilização. Propôs-se nova divisão das tarefas relacionadas ao cuidado da Sra.Júlia, ao mesmo tempo em que salientou-se a importância do uso correto das medicações e a co-responsabilização entre familiares.

Ao final dessa reunião, ficou acordado que Paula ficará responsável por verificar o uso das medicações durante os dias úteis e Pedro auxiliará no uso das medicações do final de semana, enquanto Ivete realizará a divisão das medicações semanais em potes e auxiliará quanto ao uso do glicosímetro. Por fim, tais cuidados implicaram todos os membros familiares na prestação de auxílio e promoção de mudanças de hábitos da Sra. Júlia e Sr. Luiz.

\section{Conclusões}

Podemos inferir que o uso das ferramentas de abordagem familiar propiciou o conhecimento acerca das particularidades e da dinâmica da família, permitindo assim o desenvolvimento de intervenções condizentes à realidade familiar.

Cabe aos profissisonais de saúde possibilitarem à família a compreensão do processo saúde/doença de seus membros, considerando as dimensões biopsicossociais; bem como a elucidação das possibilidades de complicações das morbidades, propiciando assim um maior engajamento dos mesmos nos planos de cuidado propostos.

Evidencia-se que a realização do estudo de família possibilitou o fortalecimento do vínculo entre os profissionais de saúde e a família assistida. Acrescenta-se que as relações de proximidade entre os profissionais e os usuários 
dos serviços de saúde favorecem a realização de cuidados integrais e continuados.

Conclui-se que a equipe de saúde da família assume importante papel junto às famílias, além de configurar-se como ponto de apoio ante os conflitos e as questões de saúde/doença.

\section{Referências}

1. Oliveira MAC, Pereira IC. Atributos essenciais da Atenção Primária e a Estratégia Saúde da Família. Rev Bras Enferm.[internet]. 2013 [citado 2019 Jan 08];66:158-164. Disponível em:

http://www.scielo.br/pdf/reben/v66nspe/v66nsp ea20.pdf

2. Souza MS, Baptista MN. Associações entre suporte familiar e saúde mental. Psicol. Argum.[internet]. 2008 [citado 2019 Jan 08];26(56):207-215. Disponível em: file:///C:/Users/User/Documents/Reisd\%C3\%AAn cia\%20R1/Doc\%C3\%AAncia/19753-34065-1-

SM.pdf

3. Mattos MA, Maruyama SAT. A experiência em família de uma pessoa com diabetes mellitus e em tratamento por hemodiálise. Rev. Eletr. Enf.[internet]. 2009 [citado 2019 Jan 08];11(4):971-981. Disponível em:

https://www.fen.ufg.br/revista/v11/n4/pdf/v11n 4a23.pdf

4. Chapadeiro CA, Andrade HYSO, Araújo MRN. A família como foco da atenção à saúde. NESCON/UFMG.[internet]. 2011 [citado 2019 Jan 18]. Disponível em: https://www.nescon.medicina.ufmg.br/bibliotec a/imagem/2726.pdf

5. Silveira Filho AD. O uso das ferramentas de saúde da família na construção do cuidado em saúde. In: ARCHANJO, D. R. Saúde da Família na atenção primária. Curitiba, Ed. IBPEX, p. 101-23, 2007.

6. Nobre LLR, Queiroz LS, Mendes PHC, Matos FV, Soares ASF, Leão CDA. Abordagem familiar no âmbito da estratégia saúde da família: uma experiência de cuidado interdisciplinar. Revista da Universidade Vale do Rio Verde. [internet]. 2014 [citado 2019 Fev 18];12(2):458$68 . \quad$ Disponível em: http://www.ruc.unimontes.br/index.php/unicien tifica/article/view/452

7. Ministério da Saúde (BR). Conselho Nacional de Saúde. Resolução n466 de dezembro de 2012. Brasília: Ministério da Saúde; 2012 [citado 2019 Fev 18]. Disponível em: http://bvsms.saude.gov.br/bvs/saudelegis/cn s/2013/res0466_12_12_2012.html
8. Ministério da Saúde (BR). Secretaria de Atenção à Saúde. Departamento de Atenção Básica. Estratégias para o cuidado da pessoa com doença crônica: hipertensão arterial sistêmica. Brasília: Ministério da Saúde; 2013 [citado 2019 Fev 18]. Disponível em: http://bvsms.saude.gov.br/bvs/publicacoes/estra tegias_cuidado_pessoa_doenca_cronica.pdf

9. Pinto SL, Silva RCR. Hipertensão arterial na infância e adolescência - prevalência no Brasil e fatores associados: uma revisão. Rev. Ciênc. Méd. Biol. [internet]. 2015[citado 2019 Fev 20];14(2):225-232. Disponível em: file://C:/Users/User/Downloads/5175-52225-1PB.pdf

10. Benicasa $M$, Tavares AL, Barbosa VMM, Lajara MP, Rezende MM, Heleno MGV, et al. The influence of relationships and alcohol use by adolescents. SMAD. [internet]. 2018[citado 2019 Fev 20];14(1): 5-11. Disponível em: http://pepsic.bvsalud.org/pdf/smad/v14n1/en_0 2.pdf

11. Jesus FA, Aguiar ACSA, Santos ALS, Meneses KF, Santos JLP. Conviving and relating to the elderly person in the household: family perception. J Nurs UFPE on line.[internet]. 2017[citado 2019 Fev 20];11(10): 4143-9. Disponível em: file://C:/Users/User/Documents/Reisd\%C3\%AAn cia\%20R1/Doc\%C3\%AAncia/231176-75164-1PB.pdf

12. Ditterich RG, Gabardo MCL, Moysés SJ. As Ferramentas de Trabalho com Famílias Utilizadas pelas Equipes de Saúde da Família de Curitiba, PR. Saúde Soc. [internet]. 2009 [citado 2019 Fev 18];18(3):515-524. Disponível em: file://C:/Users/User/Downloads/29620-

Article\%20Text-34419-1-10-20120704.pdf 13. Andrade AS, Gomes RLR. Como melhorar a capacidade de produção do colaborador utilizando as ferramentas mbti, firo-b e tki. Revista Contribuciones a la Economía. [internet]. 2018[citado 2019 Feb 20]. Disponível em: https://www.eumed.net/rev/ce/2018/1/produca o-colaborador-mbti.html

14. Moysés SJ, Silveira Filho AD. Os dizeres da boca em Curitiba: boca maldita, boqueirão, bocas saudáveis. CEBES. [internet]. 2002[citado 2019 Fev 24]: 155-60. Disponível em: http://bvsms.saude.gov.br/bvs/publicacoes/livro _curitiba.pdf

15. Lima JCM, Moraes GLA, Augusto Filho RF. O uso da conferência familiar na resolução de conflitos de uma família com idosa dependente. Rev Bras Med Fam e Com. [internet]. 2008[citado 2019 Fev 24];4(14): 5-11. Disponível em: file://C:/Users/User/Downloads/195-599-1- 
SM\%20(1).pdf

\section{Endereço para Correspondência}

Universidade Estadual de Montes Claros UNIMONTES

Av. Prof. Rui Braga, S/N - Vila Mauriceia, Montes

Claros - MG

CEP.: 39401-089

e-mail: fernandad.jesus@hotmail.com

Recebido em 16/04/2019

Aprovado em 18/11/2019

Publicado em 13/02/2020 